\title{
Cognitive Computing: Brain Tumour Detection
}

\author{
V. Dhanakoti, J. Chandru, B. Muthu Senthil
}

\begin{abstract}
The generation of the computers and the growth in this field is found to be more and more valuable to the level of abstraction of the details to the artificial intelligence and machine learning etc.., For a human the most dreadful and non-curable diseases is brain tumour. Some of the treatments and methods have been initiated, but there is no complete cure of this brain tumour. But the recent development over the computer field has brought up an idea to cure Brain Tumour.
\end{abstract}

Keywords : Cognitive science, Subdural hematoma, Glioma, Tumour cells.

\section{INTRODUCTION}

The computer to get identified itself, initiates the different ways in order to find a way for the detection of the presence of the waves in the initial stage has to be acknowledged. But the Cognitive Computer which studies about the master of the human body and its functions has to be heavily used.

Cognitive Computer is different from the normal way of computing, different way of analysing the things which were found to have more and more features that can be made over the particular things [1].

\section{COGNITIVE ANALYSIS}

Neuromorphic Computing (Or) Cognitive computing has made several levels to mimic the architecture of the brain. Since it is a complete looping of structural brain, Several Neuromorphic Cores are required to depict the architecture of the brain. So we made it to 128 neuromorphic cores for analysing the sensing of action of the brain level. Similarly different levels of abstraction of the cognitive data can be felt in the analysis of the detection of the tumour, but certain level of changes in the analysis might detects the present of the tumour [1].

\section{A. Purpose Of Cognitive Computing}

The level of the human evolution is incredible and the way it evolve in the environment has also increased the levels of development of diseases which reacts in different ways such as Dilemma, imagination, Sensational Alert [2].

Revised Manuscript Received on November 22, 2019

* Correspondence Author

Dr.V.Dhanakoti, Department of Computer Science \& Engineering, SRM Valliammai Engineering College, Chennai, India. koti555@gmail.com

Mr.J.Chnadru, Department of Computer Science \& Engineering, SRM Valliammai Engineering College, Chennai, India.

Dr.B.Muthu Senthil, Department of Computer Science \& Engineering,

SRM Valliammai Engineering College, Chennai, India.

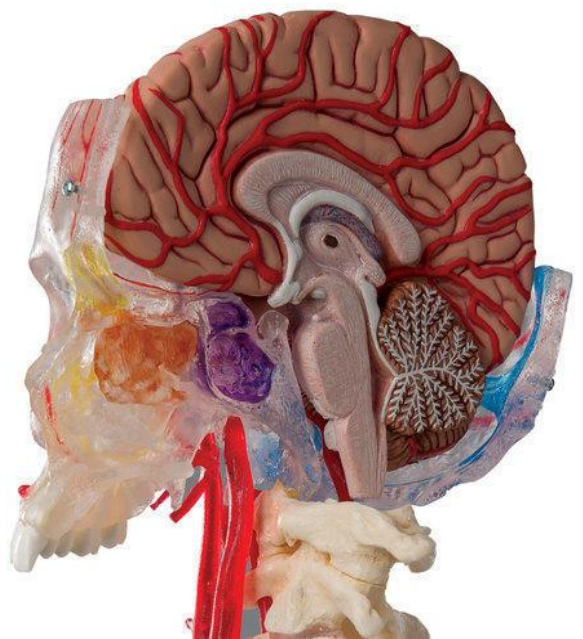

Fig1: human skull with brain model.

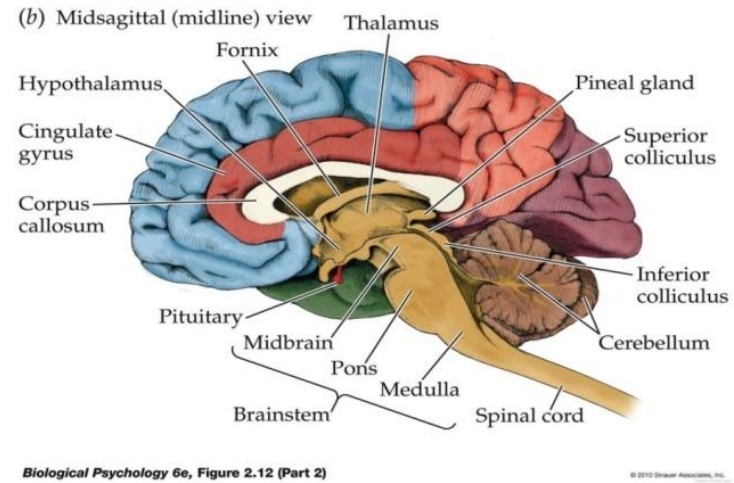

Fig2: side view of a human brain with parts.

Dreaming and generalization is due to the cognitive systems can just communicate with the brain its excel at locating knowledge, pattern identification, natural language, machine learning, eliminate bias and endless capacity[12] The newly evolved digital assistants and the digital consultants (Or) digital tutors in our present world scenarios are related to Siri and Google Assistants are not truly evolved as real cognitive systems; the present systems have been pre-programmed to the set of response. If there is certain change in the behaviour of the human as per the medical term it is called as malfunctions. But they were carried out by the brain. Cognitive Computing is configured (as technical term) in the same size of the human brain, it is structured as the helical one which strikes on the outer core of the protection layer [3]. 


\section{BRAIN MALFUNCTIONS-TUMOUR DETECTION}

A fault growth of abnormal tissue that is often uncontrolled and progressive is called cancer cells (or) tumour cells. A cancerous or non-cancerous mass in the brain which causes some sets of the abnormal conditions and faulty problems. Since it begin its growth in the brain for a while it can't be detected without the help of well-developed professionals[3].

\section{A. Brain tumour-symptoms:}

Brain tumours can cause many symptoms, but the certain levels of fatal cells can be made to be more and more vulnerable one. Some of the most common symptoms are:

- Severe Headache in the earlier session

- Changes in the ability of the person behaviours such as the way he talks which is in the abnormal way.

- The different styles of walking.

- Loss of Memory and Thinking Capability.

- Certain loss of the Muscle power and twitching [4].

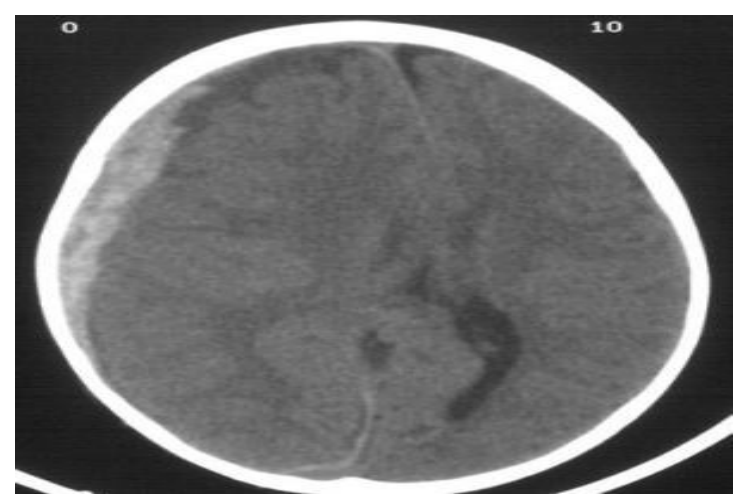

Fig:3: MRI Scan of Brain

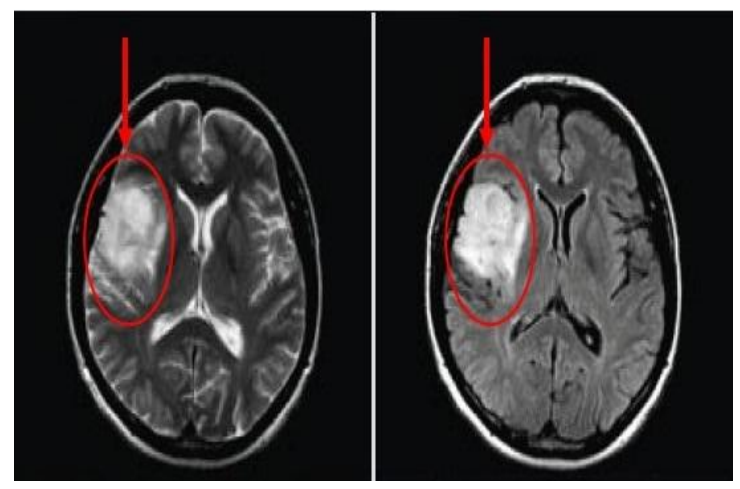

Fig-4: MRI Scan of A brain with tumour.

\section{B. Treatments and Therapies:}

In case of the brain tumour sometimes the age factor also plays a major role for the treatments [5]. Childhood: In Childhood, the transmission of diseases is carried out in the mother foetus (early growth of State). So, in the certain tumours and weird levels of the presence of cells and these dangerous cells can be a danger in order to make the system more and more acceptable one [6].

Three different and severe painful treatment are used:
- $\quad$ Surgery

- Radiation therapy

- Chemotherapy

For adults also the above treatment might be a solution for the removal of cancer cells [6].

But according to national cancer institute, Side effects after the beginning of the cancer treatment is different as data sets made to be proved which leads to the late effects as they were abnormal condition of the data sets which can't be predicted in the different levels of the system configuration which can be carried out with that cognitive computer [7].
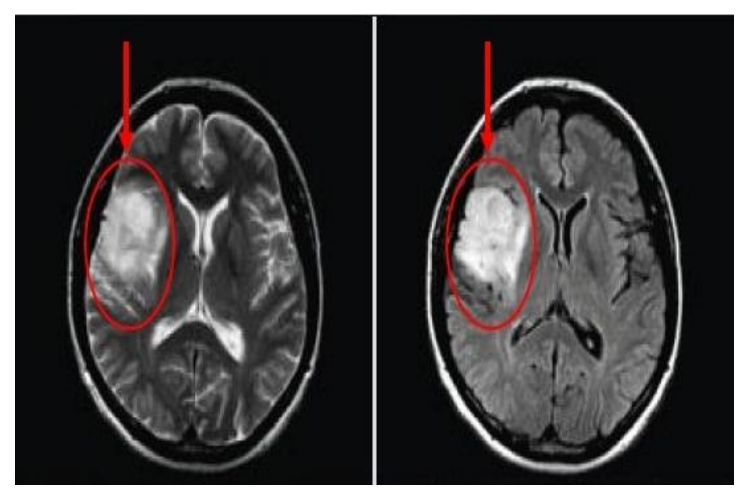

Fig 5: MRI Scan of brain with cancer.

Less awareness among the detection and effects of cancer treatment may include the following:

- Physical problems.

- Variations in mood, feelings, thinking, learning, or memory.

- New Growth of abnormal Cells (new types of cancer) [7].

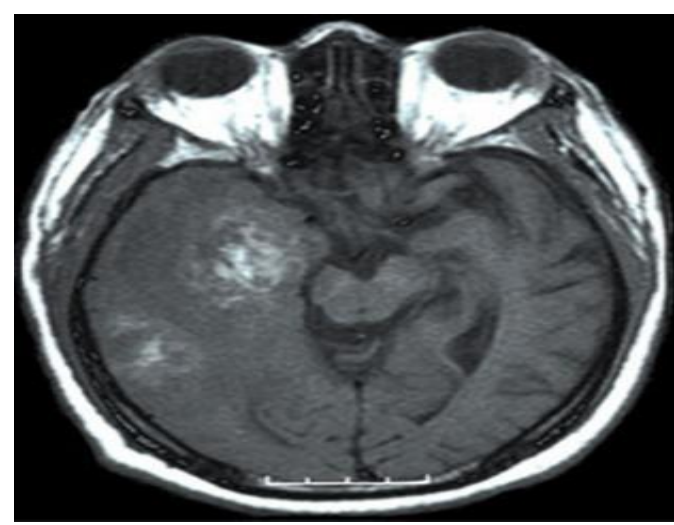

Fig-6: MRI Scan after cancer treatment

C. Computer-based assessment of cognitive functions in brain tumor patients:[6]

NeuroCogFX: a computer based test for the performance of the brain activity of the particular energized person in order to record the each and every movement of ways of the brain system [4].

NeuroCogFX is the document description of the overall function of the brain under the observation whose levels of documentation is found to be in the reduced form. NeuroCogFX

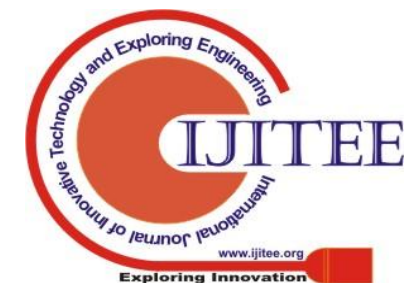


consists of the well-standardized in a group of 242 healthy controls for the detection of the presence of the Cancer levels [4].

Based on the certain level of the patient's report, if more level of the observations is found in the patients, NeuroCogFX considered as documentation of tumours and without active disease for at least 4-5 months.[8][9].

A Glioma is a most vulnerable cancer cells which starts in the body cells of the brain or the spine. The Glioma comprise about 30 percent of all brain tumours and central nervous system tumours, and 80 percent of all malignant brain tumours [10].

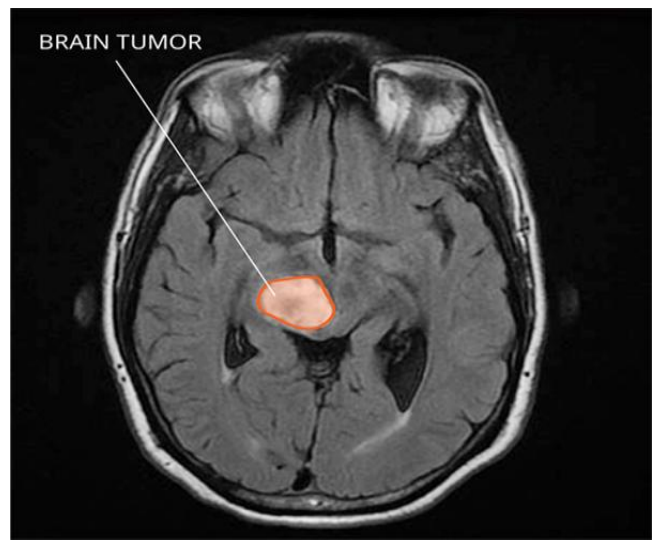

Fig 7: MRI Scan of a Brain with Glioma

So, the cognitive computing can able to detect cancer cells which is present in the brain. Since it is a one of the initiative in the medical field for the fields of the neurological science of tumour detection [2].

The computers have developed in the different versions as well as in the different forms but now it is tested to be similar to the human beings. Since there is enormous development in the fields of the Computer science, it is also extended towards the medical fields. There is less amount of treatments and analysis left in the cancer institutes and hospitals, this technique of cancer detection makes the human body to be more and more supportable to evaluate it [2].

Our System were trained to simulate human as a Computer generated Model. Using different algorithms which is in recent development that use data mining, pattern recognition and natural language processing, the computer can depicts the way the human brain works. so malfunctions can be detected at any sort of manner [6].

\section{True North's neurons to revolutionize system architecture:}

Let us assume the scenario to explain about the Truenorth that the energy consumption underscored the divergence between the brain and today's computers are well developed one which provides 100 trillon synapeses (tissues were found in the brain cells) so as per the analysis it requires that 96 blue gene/Q which stimulates 1,500 times slower than real time. A hypothetical computer to run this simulation in real-time would require $12 \mathrm{GW}$, whereas the human brain consumes merely 20W [11]

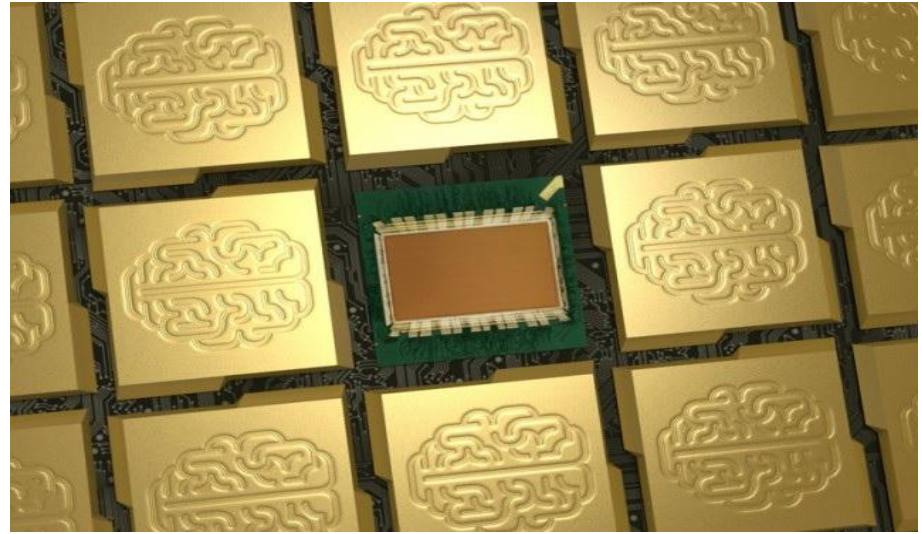

Fig 8: True north Chip

There are two factors: technology and architecture. Unlike today's inorganic silicon technology, the brain system uses biophysical, biochemical, organic wetware to alternate the performance of the different levels of the abstraction [11],

\section{E. Perspective of True North:}

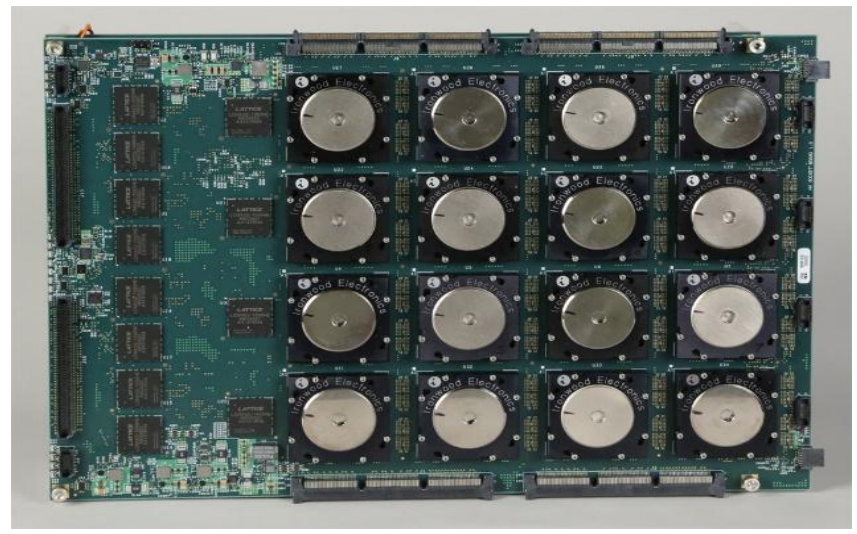

Fig 9: Top view of True North.

The general Facts of usage of the true North for this detection of tumour as it is an incredibly efficient: The chip consumes just 72 milli watts at max load, which resembles to the presence of 400 billion synaptic operations per second per watt - or about 176,000 times more efficient than a modern CPU running the same brain-like workload [12].

\section{F. Intel Loihi:}

Intel Loihi is a neuromorphic research test chip that is designed to carry asynchronous (result might be as an efficient as spikes arrives near the neuromorphic core) Spiking Neural Network (SNN) to implement adaptive one of self-modifying, event-driven fine-grained parallel computations used to implement learning and inference with high efficiency of the brain stimulation. The chip is constructed with 128-neuromorphic cores for the stimulation access of the neurons and many-core IC fabricated on $14 \mathrm{~nm}$ process and features a unique programmable microcode learning engine for on-chip [13]. 


\section{G. Neuromorphic core of Intel Loihi:}

Loihi implements of about 128 neuromorphic cores(as per the architecture of Loihi), each core is made to assign as 1,024 primitive spiking neural units(as per the analysis) grouped into tree-like structures in order to simplify the implementation[14].

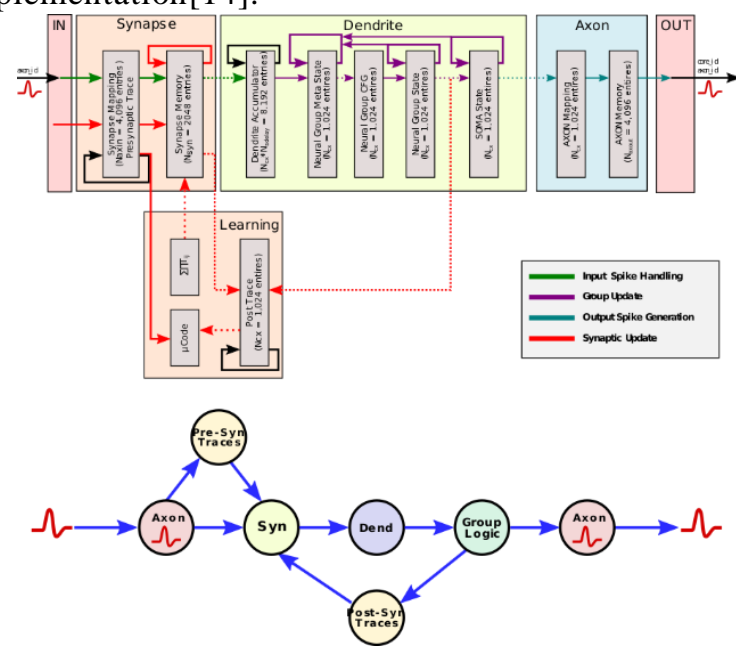

Fig 10: Major block of Neuromorphic core.

Each core were manufactured to implements of a total of 2 Mib (including ECC). Spikes are received on the input side, handled internally (synaptic/group update), and a spike is optionally generated in the output if the various conditions are met. Those blocks operate independently with little control synchronization [14]. Intel has constructed the hardware in parallelized form where a single large event can be handled more efficiently as a few smaller events. This is shown as a dotted line. One example Intel provided is extracting synapses from the memory and to the dendrite accumulator through the modification and even the learning engine is with up to four-way parallelism. In addition to the normal flow, there's the configurable learning engine shown under the major blocks [14].

\section{H. Operation with Neurons of Human Brain:}

The operation itself is fairly straightforward, once enough spikes accumulate and exceed the predefined threshold level, a spike message is created and sent out to various other groups in various destination cores[14].

Loihi is manufactured as the first neuromorphic chip to feature a fully integrated SNN meaning it can support various types of communication on the level of chip set unicast, multicast, broadcast, sparse network, variable synaptic formats (any weight precision 1-9 bits, +/-, etc..), and they were configured in the place of population-based hierarchical connectivity [14].

The iterations of all the groups by all the cores must be done within the same discrete timestamp. To ensure that all the spikes have made it to their destinations before the operation is repeated, Loihi sends out a synchronization message whereby any spikes in-flight are flushed in the first phase and in the second phase a timestep-advance notification is sent to all the cores to advance their timestamp to, enabling them to proceed to update their internal groups [14].

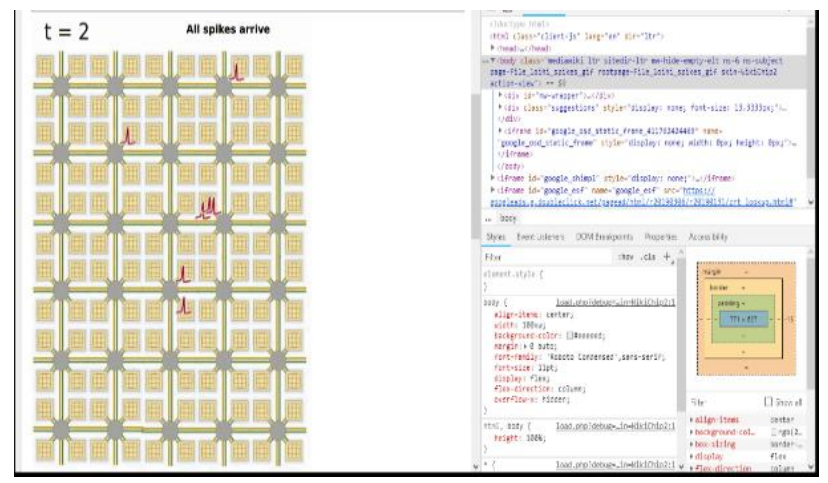

Fig 11: All neurons Arrival to the Node.

\section{Self-learning}

Each core contains a "learning engine" (marked in the block diagram above as 'learning') that can be enabled to adapt to the network parameters during operation such as the spike timings and their impact. It does this by updating the synaptic weights using the 4-bit microcode-programmed learning rules that are specifically associated with that synapse. Updates (or 'learning') is done at each learning epoch, a period of time that is globally preconfigured per core. The results of the self-learning of the chips can be configured to makes the chip more flexible as it allows various paradigms such as supervisor/non-supervisor and reinforcing/configurability without requiring any particular approach [13].

\section{J. Programming:}

We are working with tools designed by Intel for working with Loihi including a Loihi Python API, a compiler, and a set of runtime libraries were developed to build and execute SNNs on Loihi. For the most part of this work, we have used the API which is similar to existing frameworks such as Python Neural Networks. The Loihi API was designed to create a graph of neurons and synapses with certain levels of parameters such as decay time, synaptic weight, and spiking thresholds. The graphs can then be initiated by injecting external spikes. Now, it is the turn for the chip to begin to collect the information about the part which is attached in the brain loop. Now here, Spikes tells about the arrival of neuron. According to the recent details about the brain activity and its results, Here the spikes were designed as a generation of each neuron ie.; Each part generate each type of information to the particular as it travels to the neuron from the 
brain to the destination part of the organ to initiate something like Blood circulation, Hormone Regulation, Blood Pressure etc.., So this type of information can be initiated in order to Make the process in an efficient manner.

\section{RESULT AND DISCUSSION}

\section{K. Result of Brain Stimulation:}

\section{Screen Shot 1:}

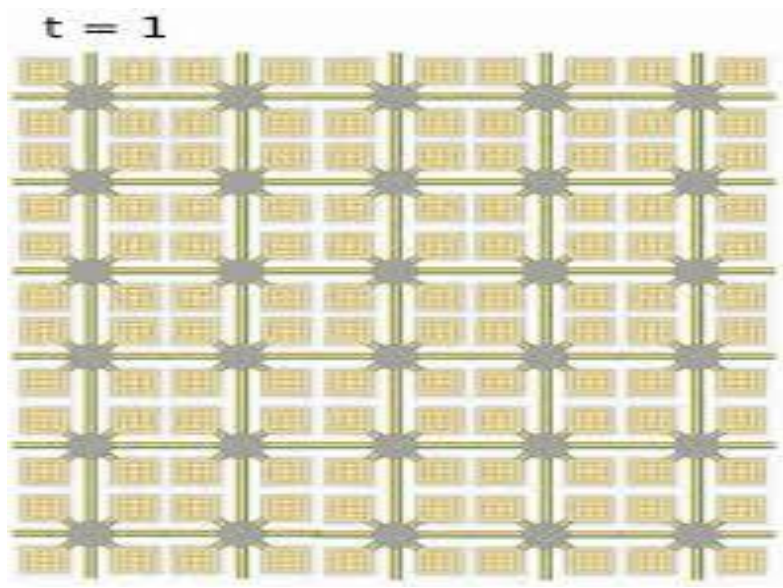

Fig 11: Structural Arrangement

Wherever the four types of routes which travel from the different parts meets, form a junction. Here the junction is considered as a Neuromorphic Core. This core carries the major part of the operations. Each junction is configured as a message synchronization. Messages can be saved at the particular Neuromorphic core along with the direction of flow of instructions.

$$
\mathrm{t}=\mathbf{1}
$$

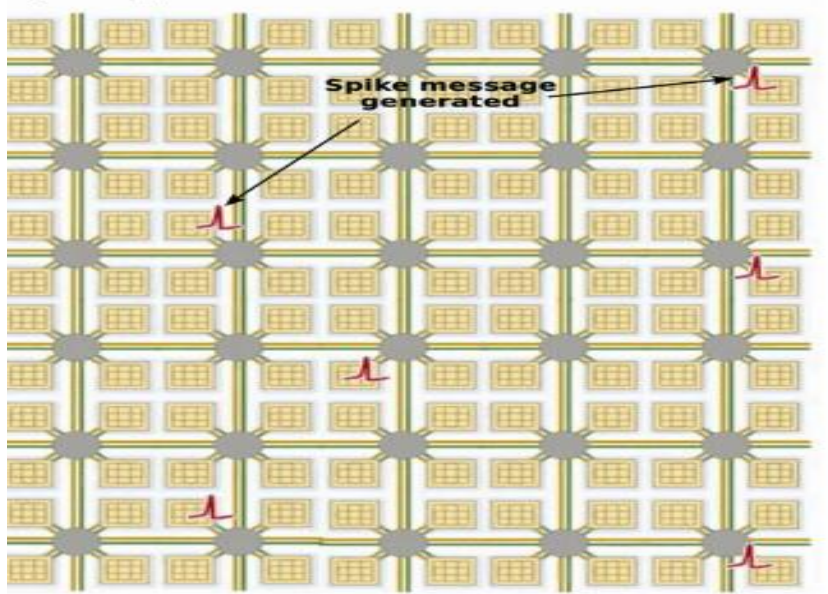

Fig 12: Neuron Spikes generation.

Now the Spikes are generated at each entrance of the Neuromorphic Core. Now the spikes resemble the generation of signals at the core. Millions of Axons are connected to form a Junction. Since it is the beginning of the transmission, " $t$ " resembles the time of the generation of the Axons and it is set to 1 .

Fig13 : Travelling of Spikes.

The information which is present in the core transfers it to all the cores in the chips. So different types of the chips were configured based upon the ability of the IQ level (or) based upon the activity by conducting the short test. The result decides whether the type of core makes to see the stimulation or detection of cancer.

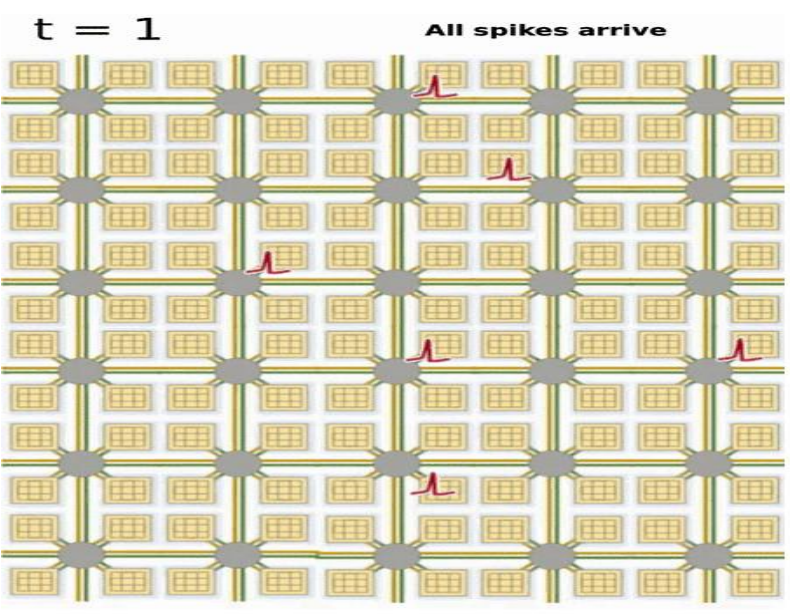

Fig14: Spikes Arrived at the Neuromorphic Core. 


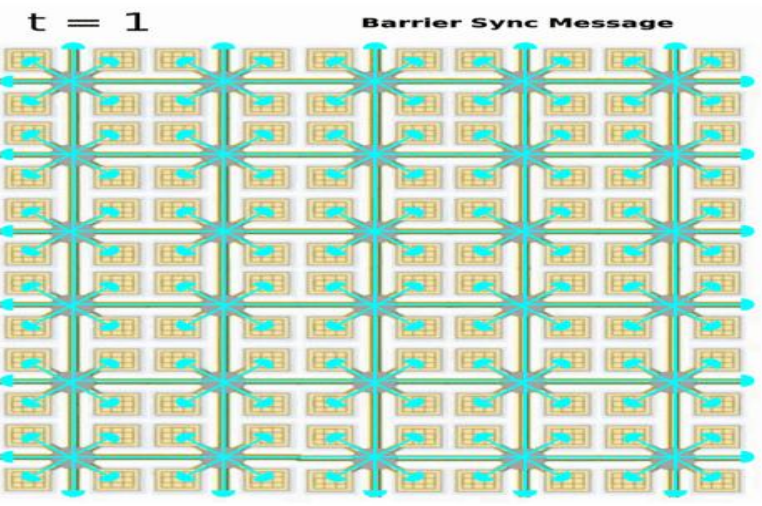

Fig15: Barrier Sync Message to all Cores

This picture tells about the complete arrival of the spikes at the time $t=1$. The spikes arrived in one of the entry point of the core. So in detection of the cancer if the spikes don't arrive at the neared cores, then some cells which prevent the transmission of instruction transfer it to all parts. So the system is configured as a presence of the cancer for the particular person

Since the arrival of spikes were present in the particular junction, all the messages will be transferred as a Barrier Sync Message to all the parts of the brain to tell information is transfer.

\section{CONCLUSION}

As computers become more able to think like human beings, they will also expand our capabilities and knowledge [5]. Just as the heroes of science fiction which has been discovered for the different levels of the prediction of data, similarly different levels of the abstraction can be performed in the chip level hardware and software interface. So we will move into an era when computers can augment human knowledge and ingenuity in entirely new ways.

\section{REFERENCES}

1. https://en.wikipedia.org/wiki/Cognitive_computing

2. https://www.cancer.org/cancer/brain-spinal-cord-tumors-adults.html

3. https://www.cancer.org/cancer.html

4. Eliza Strickland "IBM Watson Takes on the Genetics of Brain Cancer.", IEEE Spectrum, Issue. 2 , pp.21-23, 2014.

5. https://www.forbes.com/sites/bernardmarr/2016/03/23/what-everyone-sh ould-know-about-cognitive-computing\#3518fda45088.

6. Maria Stella, P.Sulman, Andrea Califano and Antonio Lavarone, "The transcriptional network for mesenchymal transformation of Brain tumours", Nature research, Vol.46, Issue.3, pp.318-325, 2009.

7. https://www.cancertherapyadvisor.com/home/cancer-topics/brain-cancer/ brain-cancer-treatment-regimens

8. Shannon 1.Scott, Chia-hsien hsu,Dina I. Tsukrov, Daniel A.Haber and Richard J.lee, "Isolation of circulating tunor cells using microverters", PNAS, Vol.2, Issue.10, 2010.

9. https://en.wikipedia.org/wiki/Neuromorphic_engineering

10. Dharmendra S. Modha, "Introducing a Brain Inspired Computers", IBM IEEE Spectrum, Vol.63, Issue.10, 2004

11. Carlos Buesa, Tamara Maees, Francesca Subirada and Marla Barrachina, "DNA chip Technology in Brain Banks: Confronting a Degrading World" , Neuropathology and Experimental Neurology, Vol.63, Issue.10, 2004

12. https://www.intel.com/content/www/us/en/research/neuromorphic-compu ting.html
13. Chetan Bettegowda, Mark Sausen, Rebecca J. Leary, Issac Kinde and Nishant Agarwal, "Circulating Tumor DNA for Early detection and managing Resistance”, Science Translational Medicine, Vol.6, Issue.224, pp.221-223, 2014

14. https://en.wikichip.org/wiki/intel/loihi.

\section{AUTHORS PROFILE}

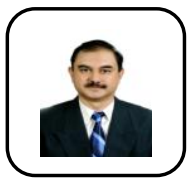

Dr. Dhanakoti V. is currently working as an Associate Professor in the Department of Computer Science and Engineering, SRM Valliammai Engineering College, Chennai. He has more than Twenty years of teaching experience. He obtained his graduate degree in Computer Science from University of Madras, Chennai and pursed his post graduate degree in Computer Science and Engineering from the year 2005. And Obtained his Doctorate in Network Security from the Anna University, Chennai, India. His research interests include Network Security, Knowledge Discovery and Data Mining, Soft Computing and Distributed Computing. He has published more than 40 research papers in International Journals and presented 45 papers in International Conferences. He has attended several seminars and workshops in the past fifteen years. He has also organized several symposiums and workshops. He has guided more than $40 \mathrm{UG}$ and PG projects. $\mathrm{He}$ is a life member of various bodies such as ISTE, Computer Society of India, etc. Currently three research Scholars are working with him.

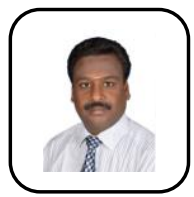

Dr. Muthusenthil Balasubramanian received the degree in Electronics \& Communication Engineering from Madras University, in 1996 Masters Degree from Satyabama University in 2007, Doctorate from Anna University, Chennai 2016. Currently, he is a Research Scientist in Wookyoung Information technology, Daegu, Southkorea and Associate Professor at SRM Valliammai Engineering College, Chennai. His interests are in mobile ad-hoc networks, network security, video security, network attacks, privacy preservation, trust evaluation and cloud computing. He is affiliated with scientific publications, he has served as invited reviewer. Further info on his homepage: http://www.srmvalliammai.ac.in. He is active Life member of CSI, ISTE and Indian Science Congress.

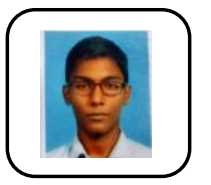

Chandru $\mathbf{J}$ is a Student of SRM Valliammai Engineering College. His research interests include Network Security, IoT and Data Mining. He has attended few conferences such as GST-A BOON OR BANE- conducted by Department of General Engineering in SRM Valliammai Engineering College and Recent Application of Computers- Conducted by Department of MCA in DR. B.S. Adbur Rahman Crescent College. Few of his projects are Target Detection Using Arduino and Automation of Speedometer Using Python. Workshop attended by him are Recent trends in computer applicationConducted by Velammal institute of Technology and Data Analytics Conducted by Computer Society of India. 